\title{
The Motivating Effects of Distraction on Task Performance
}

\author{
Glenn S. Sanders and Robert Steven Baron \\ University of Iowa
}

\begin{abstract}
Two studies were conducted to assess whether distraction has drivelike effects on task performance. In both, the effects of distraction over all trials interacted significantly with the nature of the task; distraction tended to facilitate the performances of simple tasks and significantly impaired performance on complex tasks. Moreover, analyses focusing upon drive carryover effects generally replicated these effects; when distraction was momentarily suspended, performance on simple tasks was facilitated in both studies (compared to nondistraction controls, joint $p<.05$ ), whereas performance on the complex task used in Study 2 was still impaired. Results of Study 2 indicate that lack of impairment on the complex task in Study 1 on such trials was due to practice effects of repeated testing. These results indicate that distraction (a) has drivelike properties and (b) does not invariably impair performance. These results are discussed in terms of recent research and theorizing in the area of social facilitation.
\end{abstract}

The effects of distraction upon task performance would appear to be obvious. By decreasing the amount of time and/or attention spent on the task, distraction should impair performance. There are, however, both theoretical and empirical grounds for questioning the validity of such a conclusion. Allport (1924), in his classic work on social facilitation, mentioned overcompensation as one possible explanation of superior group performance: "We work so hard to overcome the distraction incident to group activity that we actually accomplish more than we would without these hindrances" (p. 284). According to this view, the reaction to distraction is some increase in motivation, which results in a net performance improvement.

Several writers (e.g., Kimble, 1961, p. 459) have suggested that conflict, defined as competing reaction tendencies, is a source of drive. Distraction, by definition, represents such a conflict: Responses elicited by the task at hand are brought into conflict with reactions (e.g., orienting responses) to the

This article is based upon a masters thesis submitted by Glenn S. Sanders to the Graduate College of the University of Iowa. We would like to thank Charles Spiker and Nickolas Cottrell for their assistance and comments.

Requests for reprints should be sent to Glenn S. Sanders, Department of Psychology, University of Iowa, Iowa City, Iowa 52242. distracting stimulation. In this manner, distraction could increase the drive level of the performer and consequently improve performance on simple tasks (e.g., Spence, Taylor, \& Ketchel; 1956):

There is yet another, less direct, way in which distraction might increase the drive level of the performer. Freely choosing to expend high effort on a task for a minimal amount of justification should create dissonance for subjects. Such dissonance in turn could create drive effects, since dissonance is presumed to be a motivational construct with properties similar to those of drive. Indeed, a number of studies (e.g., Cottrell \& Wack, 1967) have reported drive effects on task performance when dissonance was induced in the subjects. Therefore, distraction might increase the performer's drive through dissonance induction. In accord with this reasoning, Baron, Baron, and Miller (1973) recently concluded that dissonance theory offers a tenable account of the data from research investigating why distraction increases the impact of certain persuasive messages.

Therefore, it seems possible that distraction, rather than impairing performance, might under some conditions lead to improved performance due to an increase in the individual's general motivational or drive level. The present research entails two studies, both of which test this general proposition by 
rigorously examining the effects of distraction on task performance. In the first study, subjects performed both a simple and a complex copying task (the second study essentially replicated the first, using a different set of drive-sensitive copying tasks).

The manipulation of distraction in both experiments consisted of requiring subjects, upon hearing a signal, to look away briefly from the task. In this manner, the amount of distraction experienced by the subjects was more or less completely under the control of the experimenter. ${ }^{1}$ On any given trial, subjects in the distraction condition received either $0,2,4,6$, or 8 distraction signals. It was predicted that, in general, distraction would facilitate performance on the simple task and impair performance on the complex task.

The distraction manipulation we used was selected in order to ensure that subjects would not be able to adapt to the distraction. Reim, Glass, and Singer (1971) found rapid physiological adaptation to task-irrelevant noise. Such an outcome would, of course, diminish the effects of distraction on task performance. ${ }^{2}$ We ran zero trials (i.e., no distraction delivered) in the distraction condition so that we could look more closely at the drive properties of distraction. Spence (1956, pp. 179-189) hypothesized that drive persists in the form of a covert emotional response and has presented evidence that drive carry-over occurs with delays of up to 30 minutes. If distraction produces drive, then the effects of this drive should certainly carry over onto zero-signal trials, since the intertrial interval in the present research is only $10 \mathrm{sec}$. Impairment on the complex task coupled with facilitation on the simple task during the zero trials would be particularly compelling evidence in support of a drive interpretation of distraction effects on task performance, given that on nonzero trials (or across all distraction trials), impairment on

\footnotetext{
${ }^{1}$ See Baron, Baron, and Miller (1973) for a discussion of the distinction between distraction under the control of the experimenter and distraction not so controlled.

${ }^{2}$ An additional consideration was that preliminary work indicated that "inadvertent" distractions tended to arouse suspicion.
}

complex tasks under conditions of distraction could be explained without reference to drive (in terms of required time away from the task).

\section{STUDY 1}

\section{Method}

Subjects. Subjects were 40 University of Iowa undergraduates, 8 males and 32 females, from the introductory psychology course pool. Of these, 20 subjects were randomly assigned to each of the two distraction conditions and were run individually. No subjects were eliminated from the analyses.

Design. A $2 \times 2$ factorial design was used, which varied the presence or absence of distraction on a between-subjects basis and the type of task (complex and simple) on a within-subjects basis. Subjects worked on the tasks for a total of 10 trials, each trial lasting $40 \mathrm{sec}$ with a 10-sec intertrial interval. Five trials were devoted to each of the two tasks, and these two blocks of 5 trials were alternated such that the subjects worked on a particular task on the 1st, 3 rd, 5 th, 7 th, and 9th trials and on the other task on the remaining trials. The two tasks were counterbalanced between subjects as to which task was begun on the first trial.

In the distraction condition, on any given trial the subject received either $0,2,4,6$, or 8 distraction signals. These were distributed randomly across the 10 trials, with the restriction that each had to occur exactly twice. Furthermore, averaging across all subjects in the distraction conditions, each level of distraction $(0,2,4,6$, or 8 signals) was distributed evenly across trials so that distraction level was not confounded with trial effects.

Materials. Subjects completed two copying tasks. Both presented subjects with rows of empty boxes. In the complex task, over each box was a number that had a coded alternative signified at the top of the page. The subject was to enter the appropriate alternative in each box. This complex-number task was used by Wack and Cottrell (1969). The simple task (hereafter cited as the simple-letters task) was similar, but letters were substituted for numbers in order to avoid intertask interference, and subjects simply recopied the letters that appeared without referring to the code.

Procedure. Subjects arrived for an experiment titled "Concept Formation" and were given a brief description of a concept formation task (which was never actually administered) by the male experimenter. They were then told that the copying tasks were intended to provide participants with a common preliminary experience and that while the performance on the copying tasks would not be evaluated, it was important to pay attention to them.

The experimenter then read the task instructions (see Wack \& Cottrell, 1969) and randomly assigned subjects to conditions. In the no-distraction condition, subjects began to perform the tasks immediately. In the distraction condition, the experimenter 
told subjects that to give them a particular mental orientation toward the concept formation task, he would require them to glance up at a target whenever he knocked on the desk. The target was an " $\mathrm{X}$ " on the wall in front of the subjects. Subjects were told to look at it "just long enough to get it in your line of vision." After questions, the task was begun. The $\mathrm{X}$ was $\frac{1}{2}$ inch square $\left(1.2 \mathrm{~cm}^{2}\right)$ taped on the wall approximately 3 feet $(.9 \mathrm{~m})$ in front of the subject and 3 feet $(.9 \mathrm{~m})$ above the desk. The subject had to raise his head to focus on the $X$, and the experimenter could easily determine if the instructions were being followed. The experimenter sat behind the subject and timed the trials with a stop watch. Distraction signals were delivered at irregular intervals, so that the subject could not use the between-signal interval to determine how many signals to expect on that trial. In all cases, the experimenter was blind with respect to whether the subject was working on a simple or a complex task.

After the 10th trial had been completed, the subjects were given a brief questionnaire. The subjects were then fully debriefed.

Dependent measures. The most important measure was the number of symbols correctly transcribed on each trial. ${ }^{8}$ Postperformance questionnaire material was presented in the form of 21-point scales, labeled at the extremes and at the midpoint. There were 6 such questions, and these dealt with the degree of enjoyability and interest of the tasks, the amount of distraction and tension experienced while performing the tasks, and the amount of perceived physical and psychological effort expended on the tasks.

\section{Results}

Effectiveness of the distraction manipulation. First, the experimenter observed that all subjects responded correctly to the distraction signal. Second, subjects in the distraction conditions felt more distracted than did their control condition counterparts, $F(1$, $38)=18.07, p<.01$. Finally, there was evidence that subjects attended to the distraction signal. Within the distraction conditions, there was a clear tendency for performance to be better on the zero-signal trials than on the trials where at least two signals were delivered (see Table 1 for means). The difference between the zero trials and the other distraction trials, that is, the 0 versus $2+4$ $+6+8$ comparison, was significant for the

\footnotetext{
${ }^{8}$ In both this study and the one reported below, the data were also analyzed after applying a log transformation to the data in order to minimize any variance inequalities. These analyses only strengthened the effects reported in the text.
}

simple task, $F(1,71)=4.63, p<.05$, and approached significance for the complex task, $F(1,71)=2.80, p<.10 .^{4}$ Therefore, both the performance and self-report data indicate that the manipulation of distraction was effective.

Performance scores. A $2 \times 2 \times 5$ (Task $\times$ Distraction $\times$ Trials) analysis of variance was run, using number of symbols correctly transcribed as the dependent measure. ${ }^{5}$ The task and trial main effects were highly significant: For the task effect, $F(1,38)=$ $507.48, p<.001$; for the trial effect, $F(4$, $152)=107.13, p<.001$. These findings reflect improved performance over trials and superior performance on the simple-letters task (see means in Figure 1). The Presence versus Absence of Distraction $\times$ Type of Task interaction was also significant, $F(1$, $38)=7.17, p<.05$, with distraction producing improved performance on the simple-letters task and impaired performance on the complex-numbers task. The simple effect was significant for the complex-numbers task, $t(38)=2.13, p<.05$, but not for the simpleletters task, $t(38)=1.58, .10<p<.15$.

If drive produced the interaction reported above, then because of drive carryover, a comparison of zero-trial performance with composite performance in the control condition should yield simple effects like those discussed in the preceding paragraph. This parallel obtained for the simple-letters task, with better performance on zero trials than on the composite of the control condition, $t(29)=2.23, p<.05$. However, there was no such parallel for the complex-numbers task. The means actually showed a nonsig-

\footnotetext{
4 Distraction signals were distributed such that, for example, one subject might be performing the simple task both times four signals occurred on a trial, while another could receive one four-signal trial on the simple task and the other four-signal trial on the complex task, etc. Because of this, there were less than the total sample of 20 subjects contributing data to each of the cells in Table 1 (the number of independent observations per cell is indicated in parentheses). If a subject had two scores in the same cell (e.g., if he had both four-signal trials on the simple task), the two scores were averaged and treated as an individual observation.

5 There were only two transcription errors made in the entire study, both on the simple-letters task and both by the same subject. Thus, the dependent measure is essentially an indication of response speed.
} 
TABLE 1

Mean Performance Broken Down by Number of Distraction Signals Delivered During a TRIAL IN Studies 1 and 2

\begin{tabular}{|c|c|c|c|c|c|c|}
\hline \multirow[b]{2}{*}{ Task } & \multirow{2}{*}{$\begin{array}{l}\text { No- } \\
\text { distraction } \\
\text { controls }\end{array}$} & \multicolumn{5}{|c|}{ No. distraction signals } \\
\hline & & 0 & 2 & 4 & 6 & 8 \\
\hline
\end{tabular}

Study $1^{\text {a }}$

\begin{tabular}{lrrrrrr}
\hline $\begin{array}{l}\text { Simple: Letter } \\
\text { copying }\end{array}$ & & & & & & \\
$M$ & 53.16 & 62.54 & 56.69 & $\mathbf{5 6 . 1 3}$ & 56.19 & 56.05 \\
$S D$ & $\mathbf{1 0 . 7 4}$ & $\mathbf{1 2 . 8 4}$ & $\mathbf{8 . 7 8}$ & $\mathbf{1 1 . 3 0}$ & $\mathbf{8 . 3 0}$ & $\mathbf{1 0 . 3 7}$ \\
& & & & & & \\
Complex: Number & & & & & & \\
copying & & & & & & \\
$M$ & 28.97 & 29.36 & 27.87 & 26.87 & 26.62 & 25.22 \\
$S D$ & $\mathbf{3 . 3 1}$ & 2.56 & 3.29 & 3.96 & 3.16 & 4.46 \\
& $(20)$ & $(11)$ & $(16)$ & $(15)$ & $(16)$ & $(18)$ \\
\hline
\end{tabular}

Study $2^{b}$

\begin{tabular}{lrrrrrr}
\hline $\begin{array}{l}\text { Simple: Number } \\
\text { copying }\end{array}$ & & & & & & \\
$M$ & 73.13 & 81.38 & 77.75 & 76.63 & 74.63 & 72.50 \\
$\begin{array}{l}S D \\
\text { Complex: Reverse } \\
\text { letter copying }\end{array}$ & $\mathbf{9 . 2 8}$ & $\mathbf{8 . 5 0}$ & 7.55 & 8.98 & 6.07 & 6.44 \\
$M$ & & & & & & \\
$S D$ & 28.88 & 23.79 & 21.25 & 21.63 & 20.50 & 18.38 \\
$S D$ & 4.82 & $\mathbf{3 . 2 8}$ & 6.02 & $\mathbf{5 . 2 9}$ & 6.14 & $\mathbf{5 . 6 3}$ \\
\hline
\end{tabular}

Note. In both Studies 1 and 2 all cells to the right of the no-distraction controls data within a row are related; that is, the same subjects contributed data to all levels of distraction signals for a given task.

a In Study 1, the number of independent observations per cell is in parentheses at the bottom of each column.

b Only the data for the first task is presented in this table. $n=8$ per cell.

nificant trend toward facilitation under zerotrial distraction, $t(29)<1$.

Questionnaire data. There were no significant differences between the two distraction conditions on any of the questionnaire items (all $F_{\mathrm{s}}<1.1$ ). However, this may be misleading, as several subjects reported that they felt differently about the two types of tasks, and so specific task differences between conditions may be obscured (since subjects were asked about the tasks as a whole).

\section{Discussion}

The significant interactive effect of distraction and type of task on task performance, collapsed across trials, would appear to provide support for the contention that distraction, at least as operationalized in the present study, is a source of general motivation or drive. However, the failure to obtain evidence of impaired performance on the zero trials for the complex-numbers task is clearly inconsistent with a drive interpretation of the facilitation of simple-letters task performance observed on those same trials. There are several possible explanations for this failure: First, obtained effects are not due to differences in drive level. Second, the complex-numbers task is not truly complex for the present population. And, third, it may be difficult to produce impairment when the complex task is administered after subjects have been practicing on a related task. Indeed, studies validating the drive-sensitive nature of such tasks typically employ a between-subjects design (e.g., Wack \& Cottrell, 1969). Unfortunately, since the tasks were alternated on a trial-by-trial basis in Study 1 , it was not possible to test for the presence of sequence effects. Therefore, a follow-up study was run that attempted to resolve some of the issues discussed above.

\section{Study 2}

This second study essentially replicates the previous experiment, with the following
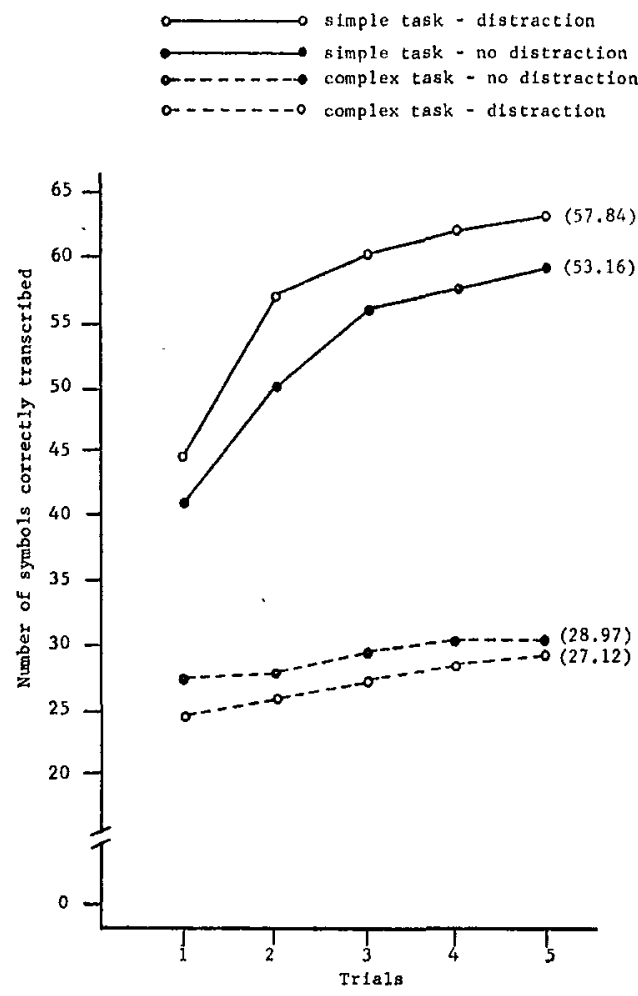

Figure 1. Mean performance scores in Study 1 (with composite means, collapsed across trials, in parentheses to the right of their respective curves). 
changes:

1. A reverse-alphabet printing task (validated as drive sensitive, e.g., Taylor \& Rechtschaffen, 1959) was substituted for the complex-numbers copying task. This task requires subjects to print the letter they see, upside down and backwards. If impairment were not obtained on this alternative task, then the drive-induction property of distraction would be called into serious question.

2. The simple-numbers copying task verified as drive sensitive by Wack and Cottrell (1969) was substituted for the (nonverified) simple-letters copying task.

3. Questionnaire items concerning task enjoyment, effort, and distraction were asked on each task separately rather than treating the two tasks as a unit.

4. The first five trials were spent on one task and the last five trials on the other task, in counterbalanced order.

\section{Method}

Subjects. Subjects were 32 undergraduates, 14 males and 18 females, at the University of Iowa who participated in the experiment in partial fulfillment of requirements for an introductory psychology course. Sixteen subjects were randomly assigned to each of the two distraction conditions and were run individually. No subjects were eliminated from the analyses.

Design. A $2 \times 2 \times 2$ factorial design was used, varying the presence or absence of distraction and order of task presentation on a between-subjects basis and varying type of task (the simple-numbers task and the complex-letters task) on a withinsubjects basis. There were again five trials for each task, with the same trial length and intertrial interval as in Study 1. In the distracted conditions, each subject experienced all five levels of distraction ( 0 , $2,4,6$, and 8 signals) on each task. This permits a standard analysis of variance for zero-trial performance. Furthermore, the zero trial always occurred on the third trial of the five-trial sequence for each task. This permits a comparison of zero-trial performance in the distraction condition with third-trial performance in the control conditions, as opposed to Study 1, in which it was necessary to compare zero-trial performance to composite control condition performance, which resulted in an unequal number of observations in the two terms involved in the comparison.

Materials. The physical structure of both tasks was the same as in the previous study, and the same letters and numbers were used.

Procedure. Subjects were run through the same procedure as in the previous study, except that in- structions for the two tasks were delivered separately, each immediately prior to the task at hand. When the second task was being explained, the subject was reminded that this was still part of the preliminary task referred to in the introductory remarks, and that in the distraction condition, the distraction signals would continue to be delivered during the second task.

Dependent measures. The performance scores used in the previous study again constituted the primary measure. Questionnaire items were again in the form of 21-point scales, labeled at the midpoint and extremes. For each task, there were items concerning enjoyment, physical and psychological effort, and distraction experienced, plus three items concerning the importance and justifiability of the present research and the subject's preception of choice regarding his participation in the experiment.

\section{Results}

Order effects. As noted, a question of major interest was whether the effect of distraction on the complex task was in some way altered by having some related activity (the simple task) precede the complex task. To address this question, the interaction between distraction and the order in which the task was performed was tested both for the simple and the complex task. This interaction was not significant for the simple task, $F(1,28)=$ .69 , but was highly significant for the complex task, $F(1,28)=10.10, p<.005$. The latter interaction (see Table 2 for means) indicates that control subjects performed better on the complex task than subjects in the distraction condition when the complex task was performed before the simple task, while the reverse was true when the complex task was performed after the simple task. This lends credence to the suggestion that in

TABLE 2

\section{Performance Scores (Collapsed Across Triats) IN STUDY 2}

\begin{tabular}{|c|c|c|c|c|}
\hline \multirow[b]{2}{*}{ Task } & \multicolumn{2}{|c|}{ Task presented first } & \multicolumn{2}{|c|}{ Task presented second } \\
\hline & $\begin{array}{l}\text { No } \\
\text { distrac- } \\
\text { tlon }\end{array}$ & $\begin{array}{c}\text { Distrac- } \\
\text { tion }\end{array}$ & $\begin{array}{l}\text { No } \\
\text { distrac- } \\
\text { tion }\end{array}$ & $\begin{array}{c}\text { Distrac- } \\
\text { tion }\end{array}$ \\
\hline \multicolumn{5}{|c|}{ Simple numbers } \\
\hline $\begin{array}{l}M \\
S D\end{array}$ & $\begin{array}{c}73.40 \mathrm{a} \\
9.28\end{array}$ & $\begin{array}{c}76.65 b \\
6.90\end{array}$ & $\begin{array}{c}73.77^{\mathrm{a}} \\
7.64\end{array}$ & $\begin{array}{c}72.17 \mathrm{~d} \\
8.99\end{array}$ \\
\hline \multicolumn{5}{|c|}{ Complex letters } \\
\hline$\stackrel{M}{S D}$ & $\begin{array}{c}26.90_{0} \\
3.78\end{array}$ & $\begin{array}{c}21.10 \mathrm{~d} \\
2.20\end{array}$ & $\begin{array}{c}23.92 \mathrm{~B} \\
6.39\end{array}$ & $\begin{array}{c}27.47 b \\
3.05\end{array}$ \\
\hline
\end{tabular}

Note. The same subjects contributed scores to all cells sharing a common subscript. $n=8$ per cell. 
Study 1 , some type of task interference effect altered the effects of distraction on the complex task. Consequently, in this study, the theoretical conclusions are based only on the data from the tasks that the subjects performed first. With regard to the self-report data, only those responses relevant to the task presented first are considered. It should be emphasized at this point, however, that whatever the effects of distraction on task performance appear to be, they are apparently not powerful enough to overcome intertask carryover effects, at least for complex tasks.

Effectiveness of the distraction manipulation. As in Study 1, all subjects in the distraction conditions looked up in the direction of the $\mathrm{X}$ whenever and only when the experimenter knocked on his desk. Subjects in the distraction conditions reported feeling more distracted than subjects in the control conditions, $F(1,28)=12.19, p<.005$. Of incidental interest is the finding that subjects in both distraction conditions reported feeling more distracted on the complex than on the simple task, $F(1,28)=9.81, p<.005$. The interaction between presence or absence of distraction and type of task for the self-report of distraction was not significant, $F(1,28)=$ $1.34, p>.20$. There is a clear trend for performance to decrease as the number of distraction signals delivered increases (see Table 1). Comparing performance on trials without distraction signals (zero trials) to performance on trials with signals $(2,4,6$, and 8 trials) yields a significant difference for the simple task, $F(1,28)=22.67, p<.01$, but not for the complex task, $F(1,28)=2.26$, $.10<p<.15$. As in study 1 , then, there are both self-report and performance data supporting the effectiveness of the distraction manipulation.

Performance scores. The mean number of symbols correctly transcribed on the first task are depicted on a trial-by-trial basis in Figure $2{ }^{\circ}$ Collapsed across trials, performance was better on the simple than on the complex task, $\quad F(1,28)=594.76, \quad p<.001$.

\footnotetext{
6 There were no differences in number of errors between conditions for either task reaching significance $(t<1)$. Since errors again constituted less than $1 \%$ of total responses, the transcription measure is essentially a measure of response speed.
}

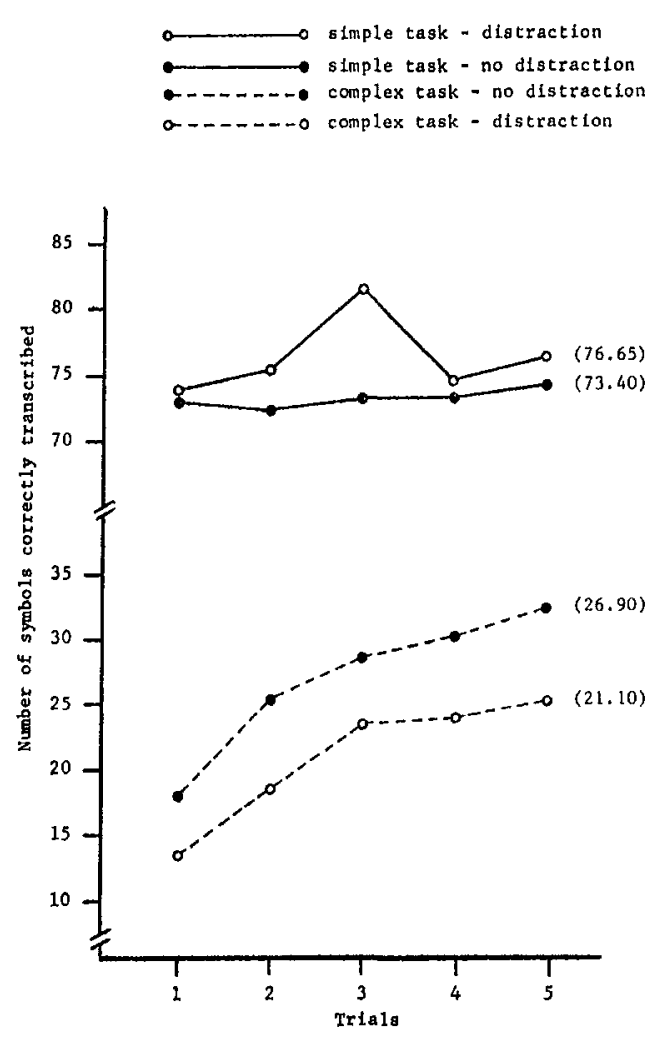

Figure 2. Mean performance scores on the first task performed in Study 2 (with composite means, collapsed across trials, in parentheses to the right of their respective curves).

There was also a significant, $F(1,28)=4.25$, $p<.05$, Presence versus Absence of Distraction $\times$ Type of Task interaction, the means indicating better performance on the simple and worse performance on the complex task in the distraction condition as compared with the no-distraction control condition. Separate analyses of the simple effects demonstrated that as in Study 1, the distractionproduced impairment on the complex task is statistically significant, $t(14)=3.74, \quad p<$ .01 , but the facilitation on the simple task is not, $t(14)=.80, p>.20$.

Subjects showed significant improvement in performance over trials, $F(4,112)=33.69$, $p<.001$, but a Task $\times$ Trials interaction, $F$ $(4,112)=18.17, p<.001$, showed that this effect was not very strong on the simple task, $F(4,56)=1.69, p>.10$, perhaps due to ceiling effects. This, of course, may account 
for why the simple effect regarding simple task performance was not more powerful.

The means of the zero trials in the distraction condition and third trial in the control condition are presented in the bottom half of Table 1. An analysis of variance shows a significant main effect for type of task, with better performance on the simple task, $F$ $(1,28)=429.52, p<.001$, and a significant Distraction $\times$ Task interaction, $F(1,28)=$ $7.40, p<.02$. Separate analyses of the simple effects demonstrate that performance was significantly worse on the complex task in the distraction than in the normal condition, $t(14)=2.45, p<.05$, while the facilitation of the simple task in the distraction condition approached significance, $t(14)=1.85$, $p<.10$. These findings are consistent with the assumption that distraction has drive properties and that this drive will carry over onto trials where no actual distraction is delivered. The significant impairment on the complex task on the zero trials in the present study contrasts with the nonsignificant trend in the opposite direction obtained in Study 1. It seems likely that the difference is due to the influences of practice and intertask interference present in Study 1. In Study 2, these influences were removed when analyzing zerotrial performance on the complex task by examining the data from only those tasks that were performed first. ${ }^{7}$

Questionnaire data. Subjects reported feeling more distracted in the distraction than in the control conditions, $F(1,28)=12.12$, $p<.005$, and on the complex than on the simple task, $F(1,28)=9.81, p<.005$, reported spending more psychological effort on the complex than on the simple task, $F(1,28)$ $11.76, p<.005$. There were no other significant differences among conditions.

7 This reasoning is strongly supported by separate analyses of variance performed on the task performed second (the right half of Table 2), both for composite scores and for zero-trial scores. Neither the main effect for distraction nor the Task $\times$ Distraction interaction reached significance for either the composite or the zero-trial measures. Moreover, as in Study 1 , there was a strong trend for distracted subjects to perform better on the complex task during the zero trial than control subjects did on their third trial.

\section{General Discussion}

To the question "Does distraction necessarily impair task performance?" the present research provides a firm "No." Even though distraction does take time and/or attention away from the task at hand (see Table 1), additional factors are apparently involved. The significant Distraction $\times$ Task interaction, collapsing across trials, in both Studies 1 and $2 \quad(p<.02$ in Study $1 ; p<.05$ in Study 2) together with the trend, though nonsignificant, for distraction (collapsed across trials) to facilitate performance on the simple task in both studies indicate that there is some compensatory process accompanying the presence of distraction. At this point, it appears quite likely that the compensatory process is in the form of an increase in the general drive level of the performer. Consistent with this conclusion is the observation of significant facilitation on the simple task under distraction on the zero trials in Study 1 $(p<.05)$ and the nearly significant facilitation in Study $2(p<.10$; joint $p$ value $<.05)$. The fact that significant impairment on the zero trials in Study 2 was observed for the complex task (when it was performed first) is further support for a drive interpretation of the effects of distraction on task performance.

Of course, this last effect could be due to factors involving distracted subjects learning the complex task less well or being distracted by anticipating a distraction signal even on zero trials. This, however, fails to explain why in both studies on the simple task, distracted subjects performed better than nondistracted subjects on zero trials. Clearly, in viewing the performance on both simple and complex tasks, across studies, the most parsimonious explanation is that distraction heightens drive.

Naturally, the case of drive induction would be stronger if the simple effects across all trials for the simple task had reached significance. However, it is important to note that in most existing work of this type, drive validation has been inferred primarily on the basis of a significant interaction in the absence of significant simple effects (e.g., Spence et al., 1956). In short, the present results are at least as strong as those of 
similar published studies, even not considering the zero-trial data.

The fact that distraction can heighten performance under certain conditions is a provocative empirical finding that contradicts commonsense expectations. Moreover, these data bring forth an interesting theoretical issue: What is the mechanism responsible for the drive properties of distraction? To the three mechanisms discussed in the introduction-an attitude of overcompensation, or "trying harder," response conflict, and dissonance induction - a fourth may be added here: uncertainty. Since subjects did not know when to expect a distraction signal, there was an element of uncertainty present in the distraction condition that was lacking in the control condition. Averill (1973) reviewed findings indicating that the reduction of uncertainty is positively related to behavioral and physiological indices of stress reduction. Therefore, it is conceivable that the increased uncertainty in the distraction condition may have led to greater stress, which in turn produced drivelike effects observed in the present research.

Whatever the mediating mechanism is, the fact that distraction appears to have drive properties has immediate relevance to research in the area of social facilitation, that is, research indicating that the presence of species mates improves dominant responses and impairs subordinate ones. This is because it is obvious that species mates may be distracting. ${ }^{8}$ Indeed, Zajonc (1965) argued that social facilitation effects may be due to drive

\footnotetext{
${ }^{8}$ Pessin (1933), in fact, found that mechanical distraction and the presence of a spectator produced very similar performance effects.
}

produced by the presence of species mates. What our findings add to this position is that social facilitation effects may be merely a subcategory of a more general phenomenon involving the motivational effects of distraction.

\section{REFERENCES}

Allport, F. Social psychology. Boston: Houghton Mifflin, 1924.

Averill, J. R. Personal control over aversive stimuli and its relationship to stress. Psychological Bulletin, 1973, 80, 286-303.

Baron, R. S., Baron, P. H., \& Miller, N. The relation between distraction and persuasion. Psychological Bulletin, 1973, 80, 310-323.

Cottrell, N. B., \& Wack, D. The energizing effect of cognitive dissonance upon dominant and subordinate responses. Journal of Personality and Social Psychology, 1967, 6, 132-138.

Kimble, G. A. Hilgard and Marquis' Conditioning and learning (2nd ed.). New York: AppletonCentury-Crofts, 1961.

Pessin, J. The comparative effects of social and mechanical stimulation on memorizing. American Journal of Psychology, 1933, 45, 263-270.

Reim, B., Glass, G. C., \& Singer, J. E. Behavioral consequences of exposure to uncontrollable and unpredictable noise. Journal of Applied Social Psychology, 1971, 1, 44-56.

Spence, K. W. Behavior theory and conditioning. New Haven, Conn.: Yale University Press, 1956.

Spence, K. W., Taylor, J, \& Ketchel, R. Anxiety (drive) level and degree of competition in pairedassociates learning. Journal of Experimental Psychology, 1956, 52, 306-310.

Taylor, J. A., \& Rechtschaffen, A. Manifest anxiety and reversed alphabet printing. Journal of $A b$ normal and Social Psychology, 1959, 58, 221-224.

Wack, D. L., \& Cottrell, N. B. Interactive effect of drive and $S-R$ compatibility on speed of digit coding. Journal of Experimental Psychology, 1969, $80,562-564$.

Zajonc, R. B. Social facilitation. Science, 1965, $149,269-274$.

(Received October 7, 1974) 